\title{
Building GIS Applications using Spatial Network Data Models
}

\author{
Folami Gbenga Obokhai \\ 14, Ajoke Dosumu Street \\ Surulere, Lagos, Nigeria
}

\begin{abstract}
The mundane tasks of Geographical Information Systems (GIS) are network modeling, network management and network analysis. Network analysis include computation of paths (routing) in transportation networks (after evaluation with markov chains e.t.c) allocation of resources' in decisionmaking and applications management. This paper is presenting a spatial network data model, a feature that allows modeling and analysis of networks; in focus on application logic. This model uses open generic data models with many common GIS capacities.
\end{abstract}

\section{General Terms}

Application Design, GIS Systems, GIS Systems Application Design, Travelling Salesman Algorithm, Accessibility Analysis Algorithm, Spanning Tree Protocol, Nearest Neighbor Analysis Algorithm, Shortest Route Algorithm, Etc

\section{Keywords}

GIS Applications, Network Spartial Data, GIS Systems Design.

\section{INTRODUCTION}

(GIS) Geographical Information Systems applications are of two basic common types:

- Applications that deal with spatial proximity.

- Applications that deal with both spatial proximity and connectivity.

These GIS applications are networks that require modeling and analysis on object relationships; object relationships refer to connectivity. Spatial Proximity- enumerates metrics like Euclidean or geodetic distances. The set of indexes, operators, and functions are made available for the managing of information based on spatial proximity; this network model however extends this framework to deal with connectivity. Consider figures $1 \& 2$; • Figure I - shows a road network of new-York city. - Figure II- shows a road network of Lagos. Issues from Common GIS vendor Network Solutions:

a) Data model is stored on proprietary file formats, and cannot be integrated with their database.

b) The Data model and Data analysis capabilities cannot be extended.

c) The problem of separating application information from connectivity information.

d) The problem of not directly supporting spatial information management and hierarchical relationships.

The framework presented by this paper addresses these issues thus:
I. Provision of an open and persistent network data model. This is achieved when network data model is stored on relational tables in the database and is managed through SQL or relevant programming language.

II. Simplification of network data management and analysis- APIs are provided for network data management and analysis. III. Separating Connectivity and application information in the data model.

III. Allowing the extension of data models and the data analysis capabilities. Only connectivity information is required for network analysis, connectivity information and application information are managed in the database.

The APIs provides representation of network elements as interfaces (e.g. Java interfaces). Users are allowed to define their own network elements by extending these interfaces; thus users can implement their own user-defined representations and analysis functions.

This paper is organized thus:

It presents the network data model database schema, APIs and architecture; shows how to use the data model; presents several GIS network applications built on top of the data model; discusses how to use network constraints to enhance analysis capabilities; discusses network data model editor that is used to edit and visualize networks.

Spatial Network Data Model: The network data model (especially the type under consideration) consists of two parts: Network Schema $\bullet$ Network APIs

Network Schema: this is the sequence of the continuous data storage used to store network information.

Network APIs: contain the relevant Sql package (programming language package) for data management in the database and java or php API (or interface) for data management and the analysis on the client side.

\section{NETWORK DATA MODEL SCHEMA:}

A network contains the following:

- Network metadata $\bullet$ A node table $\bullet$ A link (arch-node) table.

In congruence with path information, (which consists of pathtable and path -link table) can be stored with the network if desired. We would use figure III to illustrate a schematic view of a network in the database: 


\subsection{Network metadata:}

This is a concept that provides general information about networks. It includes the following details: I. Either Directed or Undirected. II. Either Logical or Spatial. III. Either Hierarchical or Flat. IV. Information about nodes, links and paths. V. Geometry information- for the case of spatial networks.

\subsection{Network Tables}

A network (especially the type under our analytical framework) contains basically two tables:

- A node table

- A Link (arch-node) table

Depending on the state of necessity and variability of data, a path table can be added. The Schema presents the information necessary for network management and analysis. Application attributes can be added to these tables or referenced from other application tables (through foreign keys). The Network data Model is also capable of handling geometric information describing that network data model can represent both logical and spatial network applications; the adding of geometric data to a logical network will allow the logical network to be displayed.

\section{SPATIAL NETWORK DATA MODEL} APIs:

The Network Data Model provides an API and a Java or Php API for network management on the database and client sides. The JAVA can also be used for network analysis.

Network Data Model Analysis Capabilities:

Network Data Model supports the following analysis methods:

- Shortest Path: this refers to the shortest path from node A to node B.

- Accessibility Analysis: is Node A accessible to node B? And if so how?

- Minimum-Cost Spanning Tree: what is the minimumcost tree that connects all nodes?

- Within Cost Analysis: What nodes are within a given cost from (to) a given node?

- Nearest Neighbors: What is the $\mathrm{N}$ nearest neighbors of a given node $\mathrm{A}$ to node $\mathrm{B}$ ?

- Connected Components Analysis: labels connected with disparaging IDs.

- K-shortest Paths: what are the K shortest paths from node $\mathrm{A}$ to node $\mathrm{B}$ ?

- Graphic Operations: Graph union, intersection and difference.

- Travelling Salesman Problem: What is the minimumcost tour that visits a set of cities?

- Single Source, Single Sink Maximum Flow Analysis: what is maximum allowable flow than can flow from the source node to the sink node?
3.1 Exert on modeling network applications

Network data Model uses a generic approach to solving network problems by specifically separating connectivity information from application specific information. First Network Connectivity information (node connection and link cost) is extracted and separated from the application-specific information. Application -specific attributes are stored, if needed with the connectivity information or separately. Once the information is extracted, network analysis is conducted on the generic model. The final result is mapped to applicationrelated attributes and displayed after additional network constraints have been also considered. This approach voids customized network solutions and simplifies data management of connectivity and application specific information.

\section{DATA STRUCTURE REPRESENTATIONS OF NETWORK} ELEMENTS:

The either Java or Php representations (network, nodes, links and paths) are defined as either JAVA or PHP interfaces (depending on specifities) and can therefore be extended. We allow these interfaces specify the necessary behaviors for the network and its elements. In addition to these interfaces userdefined analysis functions can be used by applications, allowing the modeling and analysis capabilities of the network data model to be extended.

\subsection{Using the network model}

The following are major steps:

1. Network Creation:

- Create and populate network tables and add metadata to the database.

- Create a Java or Php network object using Java or Php API and save it to the database.

2. Edit the Network:

- Load a network from the database.

- Edit the either Java or Php network object using the network data model editor; then store the network in the database, if needed.

3. Analyze the Network:

- Load a network into either the Java or Php network object (depending on specifities).

- Conduct network analysis. Make sure you save the path results especially if needed.

\subsection{Network Data Model Editor}

The Network data model editor is a standalone Php or Java application that helps create, edit and visualize networks, with extensive capabilities that can handle web solutions with adequate security. The editor aids viewing operations such as pan elements. All analysis supported, in the editor. The editor allows users to be able to create a network from scratch in the client- side and save it to the database. The editor is configurable on elements styles, colors and sizes. 


\section{GIS APPLICATIONS USING THE NETWORK DATA MODEL:}

GIS network analysis should include:

- Network tracing.

- Network routing

- Network allocation.

\subsection{Network tracing applications:}

Tracing applications deals with queries like:

- Reach ability: what nodes are reachable or can be reached from a given node?

- Connect ability: this refers to how many connected components are available in the network.

- Accessibility: what nodes are assessable to the node?

\subsection{Network routing applications:}

- Traveling Salesman Problem: find a minimum-cost tour that passes a set of locations and the minimumcost tour that visits a set of cities.

- K shortest Paths: what are the shortest paths from node $\mathrm{A}$ to node $\mathrm{B}$ ? Indicating the finding of $\mathrm{K}$ shortest paths from a start node to a destination node.

- Shortest Path or fastest Path: transitive closure problem.

\subsection{Analyze the network:}

- Load the network into either the Java or Php network object (depending on specifities).

- Conduct network analysis: make sure you save the path results especially if needed.

\subsection{Network allocation applications:}

This reveals allocation analysis which deals with destination points within a network. This provides information on a service area or coverage for points of interest.

Network data model supports the following allocation analyses:

- Within Cost: locate all points of interest within a certain distance from a designated location. Nearest Neighbor: Locate the $\mathrm{N}$ nearest restaurants to a designated location or point. - Minimum-Cost Spanning Tree: Locating the cheapest way to connect all the nodes.

\section{USING NETWORK CONSTRAINTS IN ANALYSIS:}

Constraints represent stipulated conditions to be satisfied during analysis. The network data model supports network constraints so that applications can impose application specific conditions on the network during analysis. The Java or Php interface Network constraint can be implemented by the user, and passed into any network data model analysis function.

Path Links - WHAT ARE THE LINKS TO THIS PATH EITHER SHORTEST OR

\section{ACCESSIBILITY ANALYSIS Path}

Nodes - NODAL ANALYSIS UNDER SPANNING TREE OR NEAREST NEIGHBOR ANALYSIS

Path Depth- DEPTH ANALYSIS TRAVELLING SALESMAN PROBLEM WITH ADDITIONAL INPUT

Path Cost - WITHIN COST ANALYSIS

The following are disparaging examples of network constraints:

\subsection{Turn Restrictions}

A Turn restriction refers to constraints involving two links. Their application is very common in routing connected with transportation networks. The example below, illustrates a prohibited turn represented by a start link and an end link. Considering the case for intersections with turn restrictions, such as no "U" turn or no left turn, the search might ( a case of "if")encounter the start link of prohibited turn, the search does not continue through the end link of that prohibited turn. This type of restriction can be easily modeled using network constraint, since information on the current link and the next link is made available to respective users. The directionality of a link can be further specified at the link level; unlike the network directionality that determines the directions of all links, a directed network can have links that are directed or bidirected. It would assist analysis to add a BI-DIRECTED column to the link table and use it to indicate if a directed link is bi-directed. This is an enhancement that would reduce the storage requirements for directed networks with nonhomogeneous link directions (either unidirectional or bidirectional).

Start link $=>$ End link: N1->E2 N1->W1 S2->N1 Turn type Right Turn Right Turn U- Turn

\section{NETWORK ANALYSIS: MAXIMUM FLOW ANALYSIS}

We are considering here a single source and a single sink flow network. The maximum flow analysis function is provided for a single source and single sink flow network. Each link in a flow network has a flow capacity associated with it. The goal of this function is to find the maximum allowable flow that can flow from the source node to the sink node. This analysis helps when handling logistics network planning and also has mechanical flow pattern applications. 


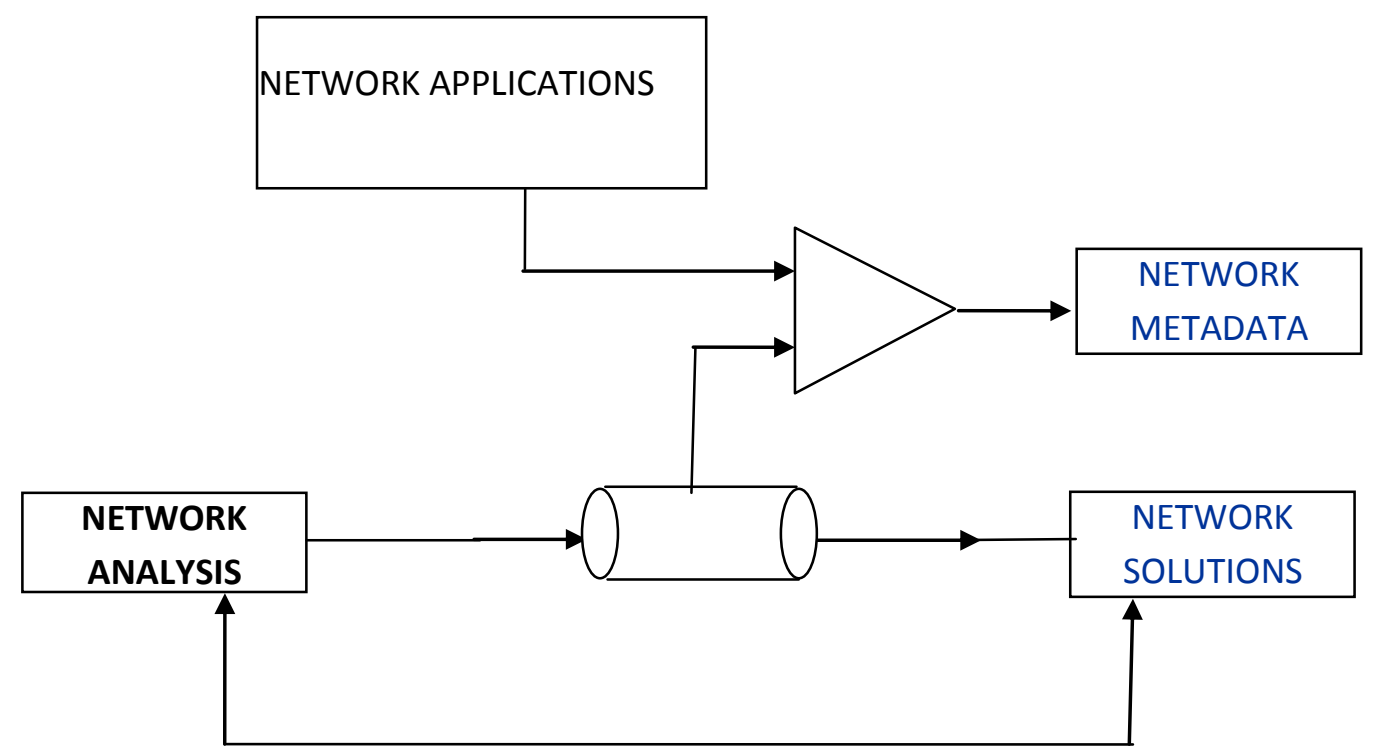

Fig 1: Integration Model For Network Applications

\section{ACKNOWLEDGMENTS}

Our thanks to the experts who have contributed towards development of the template.

\section{REFERENCES}

[1] Cantrell R.S./ Cosner C.,

[2] Spatial Ecology via Reaction-Diffusion Equations, Wiley \& Sons, 428p, 2003 Christakos G./

[3] Bogaert P./ Serre M.,

[4] Temporal GIS, Springer, Berlin, 217p, 2002

[5] Chwif L./ Barretto M.R.P.,

[6] On simulation model complexity, in: Joines J.A./ Barton R.R./ Kang K./ Fishwick P.A., Proc. 2000 Winter Simulation Conf., Orlano (Fl), 449-455, 2000 Constanza R./ Sklar F.H., Articulation, accuracy and effectiveness of mathematical models: a review of freshwater wetland applications, Ecological Modeling, Vol. 27, 45-69, 1985 Deaton M.L./ Winebrake J.I., Dynamic Modeling of Environmental Systems, Springer, New York, 194p, 1999 Finlayson B.A., Introduction to Chemical Engineering Computing, Wiley \& Sons, 339p, 2006 Gander W./ Hrebicek J., Solving Problems in Scientific Computing using MAPLE and MATLAB,

[7] Springer Publ., Heidelberg, 412p, 1997 Harper M., Sediment Nitrogen and Phosphorus Processes Model (SNAPP): User Guide, 10p,

[8] 2000 Hornberger G./ Wiberg P.,

[9] Numerical Methods in Hydrological Sciences, Am. Geophys. Union, Washington D.C., e-book, 2005. Boudreau B.P.,
[10] The diffusive tortuosity of fine-grained unlithified sediments,

[11] Geochim. Cosmochim. Acta, Vol. 60, No. 16, 31393142, 1996 Carman, P.C.,

[12] Fluid flow through porous rock, Trans. Inst. Chem. Eng. London, Vol. 15, 150-157, 1937 Carman P.C., Flow of Gases through Porous Media, Butterworths Scient. Publ., London, 180p, 1956 Drewer J., The Geochemistry of Natural Waters, Prentice-Hall, Upper-Saddle River (NJ), 436p, 1997 H“afner F./ Sames D./ Voigt H.-D., W"armeund Stofftransport, Springer Verlag, Berlin, 626p, 1992 Iversen N./ Jorgensen B.B., Diffusion coefficients of sulfate and methane in marine sediments: influence of porosity, Geochim. Cosmochim.

[13] Acta, Vol. 57, 571-578, 1993 Lide D.R. (ed.) Handbook of Chemistry and Physics, CRC Press, Boca Raton, 76th edition, 1995

[14] Luckner L./ Schestakow W.M., Migrationsprozesse im Boden- und Grundwasserbereich, Deutscher Verlag f ur Grundstoffindustrie, Leipzig, 1986 Sahimi M.,

[15] Flow phenomena in rocks: from continuum models to fractals, percolation, cellular automata, and simulated annealing, Rev. Mod. Phys., 1394-1534, Vol. 65, No. 4, 1993 Schr"oter J.

[16] Mikro- und Makrodispersivit"at por"oser Grundwasserleiter,

[17] Meyniana 36, 1-34, 1984 Weissberg H.,

[18]Effective diffusion coefficients in porous media, J. Appl. Phys., Vol. 34, 2636-2639, 1963. 